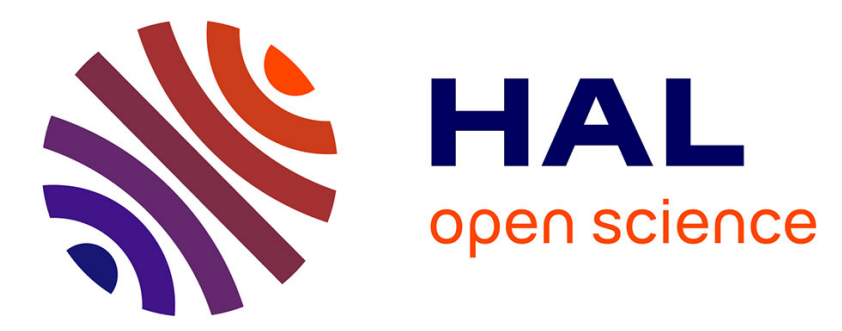

\title{
Elaboration de spatio-cartes sédimentologiques et morphologiques en milieu littoral à partir de données satellitales : un exemple en baie du Mont Saint-Michel
}

Yvette Vaguet-Marchand

\section{- To cite this version:}

Yvette Vaguet-Marchand. Elaboration de spatio-cartes sédimentologiques et morphologiques en milieu littoral à partir de données satellitales : un exemple en baie du Mont Saint-Michel. Norois, 1998, 177 (1), pp.51-62. 10.3406/noroi.1998.6848 . hal-01853867

\section{HAL Id: hal-01853867 https://hal.science/hal-01853867}

Submitted on 5 Aug 2018

HAL is a multi-disciplinary open access archive for the deposit and dissemination of scientific research documents, whether they are published or not. The documents may come from teaching and research institutions in France or abroad, or from public or private research centers.
L'archive ouverte pluridisciplinaire HAL, est destinée au dépôt et à la diffusion de documents scientifiques de niveau recherche, publiés ou non, émanant des établissements d'enseignement et de recherche français ou étrangers, des laboratoires publics ou privés. 


\section{Elaboration de spatio-cartes sédimentologiques et morphologiques} en milieu littoral à partir de données satellitales : un exemple en baie du Mont Saint-Michel

Yvette Marchand

\section{Citer ce document / Cite this document :}

Marchand Yvette. Elaboration de spatio-cartes sédimentologiques et morphologiques en milieu littoral à partir de données satellitales : un exemple en baie du Mont Saint-Michel. In: Norois, n¹77, Janvier-Mars 1998. pp. 51-62;

doi : https://doi.org/10.3406/noroi.1998.6848

https://www.persee.fr/doc/noroi_0029-182x_1998_num_177_1_6848

Fichier pdf généré le 01/05/2018 


\title{
Résumé
}

Le choix d'une donnée satellitale et d'une méthode de traitement d'image est conditionné par l'objectif de l'utilisateur. Cet article présente, rapidement, dans un premier temps, les trois méthodes les plus répandues. Les classifications permettent de regrouper les pixels semblables afin d'obtenir une image synthétique (I-A). Alors que les détections de gradients, automatiques ou non (photointerprétation), conservent uniquement les discontinuités de l'image (I-B). Quant aux modèles, ils sont I 'expression mathématique de la relation entre la réflectance et le phénomène étudié, laquelle s'appuie sur plus ou moins de mesures in situ (I-C). Dans un second temps, des exemples concrets illustreront l'utilisation de ces trois méthodes, sur des données de capteurs différents, pour l'élaboration de spatio-cartes sédimentologiques et morphologiques. Un exemple détaillé concerne une classification non supervisée d'une donnée Spot de la baie du Mont-Saint- Michel. II présente les étapes du traitement et finalement une spatio-carte des sédiments et de la végétation de la baie (II-A). La détection de gradients de la surface de l'eau permet d'appréhender la morphologie des fonds (II-B). Tandis que pour obtenir des valeurs absolues de la variable (ici la bathymétrie), il faut avoir recours à des modèles (II-C).

\begin{abstract}
Choice of a given kind of satellite data and of a given processing method depends on the goal of the user. Among the main satellite image processing methods, the classifications group the pixels in classes to made a synthetic image (I-A). Automatic gradient detection or photo-interpretation preserve only the edges in the image (I-B). Models are mathematic expression of the relation between the reflectance and the object of the study $(\mathrm{I}-\mathrm{C})$. This relation is based on a lot or few measurements on the ground. For mapping in the field of coastal morphology, this three methods and data of different satellites can be used. An example, about the Mont-Saint-Michel bay 's, explains, step by step, an unsupervised classification for mapping sediments and vegetation cover (II-A). The relative subtidal morphology can be mapped by gradient detection (II-B). While a model gives the absolutes values: bathymetry (II-C).
\end{abstract}


Norois, 1998, Poitiers, t. 45, $\mathrm{n}^{\circ} 177$, p. 51-62.

\title{
Élaboration de spatio-cartes sédimentologiques et morphologiques en milieu littoral à partir de données satellitales : un exemple en baie du Mont Saint-Michel
}

par Yvette MARCHAND

\author{
BRGM - URA 1694 du CNRS \\ Géomorphologie et transferts de surface \\ Université de Caen \\ 24, rue des Tilleuls, 14000 Caen
}

\begin{abstract}
RÉSUMÉ
Le choix d'une donnée satellitale et d'une méthode de traitement d'image est conditionné par l'objectif de l'utilisateur. Cet article présente, rapidement, dans un premier temps, les trois méthodes les plus répandues. Les classifications permettent de regrouper les pixels semblables afin d'obtenir une image synthétique (I-A). Alors que les détections de gradients, automatiques ou non (photointerprétation), conservent uniquement les discontinuités de l'image (I-B). Quant aux modèles, ils sont l'expression mathématique de la relation entre la réflectance et le phénomène étudié, laquelle s'appuie sur plus ou moins de mesures in situ (I-C). Dans un second temps, des exemples concrets illustreront l'utilisation de ces trois méthodes, sur des données de capteurs différents, pour l'élaboration de spatio-cartes sédimentologiques et morphologiques. Un exemple détaillé concerne une classification non supervisée d'une donnée Spot de la haie du Mont-SaintMichel. Il présente les étapes du traitement et finalement une spatio-carte des sédiments et de la végétation de la baie (II-A). La détection de gradients de la surface de l'eau permet d'appréhender la morphologie des fonds (II-B). Tandis que pour obtenir des valeurs absolues de la variable (ici la bathymétrie), il faut avoir recours à des modèles (II-C).
\end{abstract}

\section{ABSTRACT}

Choice of a given kind of satellite data and of a given processing method depends on the goal of the user. Among the main satellite image processing methods, the classifications group the pixels in classes to made a synthetic image (I-A). Automatic gradient detection or photo-interpretation preserve only the edges in the image (I-B). Models are mathematic expression of the relation between the reflectance and the object of the study $(I-C)$. This relation is based on a lot or few measurements on the ground. For mapping in the field of coastal morphology, this three methods and data of different satellites can be used. An example, about the Mont-Saint-Michel bay's, explains, step by step, an unsupervised classification for mapping sediments and vegetation cover $(I I-A)$. The relative subtidal morphology can be mapped by gradient detection (II-B). While a model gives the absolutes values : bathymetry $(I I-C)$.

Mots-clés : Morphologie littorale. Télédétection. Cartographie.

Key words : Coastal morphology. Remote sensing. Mapping. 
La télédétection satellitale apparue dans les années 70 continue de se développer tant par le lancement de nouveaux capteurs (ERS, JERS) que par le perfectionnnement des techniques de traitement des données. Mais le choix de telle donnée satellitale et de telle méthode de traitement d'image est conditionné par l'objectif thématique. Cet article se propose de faire le point dans le domaine de la géomorphologie littorale. Dans un premier temps, une recherche bibliographique présente les grandes lignes des trois principales familles de méthodes de traitement d'image satellitale que constituent les classifications, les détections de gradients et les modèles. Dans un second temps, un exemple de classification en baie du Mont-Saint-Michel est détaillé. Ft les autres méthodes sont aussi illustrées par des cas concrets.

\section{I. - PRÉsentation des mÉthodes}

\section{A) LES CLASSIFICATIONS}

Les méthodes de classification sont nombreuses et ont été répertoriées dans leur majorité par l'équipe FRALIT (1977). Il est possible d'en distinguer deux grands types : les classifications supervisées et non supervisées. Les pixels sont regroupés en classes homogènes dont les centres initiaux sont donnés par l'opérateur à partir de zones d'apprentissage extraites de l'image (supervisée), ou tirés aléatoirement (non supervisée). Le premier cas implique une connaissance préalable du terrain tandis que dans le second cas l'utilisateur doit retrouver la taxonomie à partir des signatures spectrales des barycentres. Le critère usuel d'agrégation consiste à minimiser l'inertie intraclasse et à maximiser l'inertie interclasse. Toutefois, il existe une infinité de variantes selon, notamment, le type de distance choisie. Les classifications, supervisées ou non, sont des outils largement répandus y compris dans le domaine littoral.

Le choix des attributs sur lesquels la classification s'établit, est issu d'une réflexion à l'amont. La corrélation entre l'image résultante et la réalité, du point de vue de la thématique choisie, est d'autant plus forte que les attributs retenus traduisent bien le phénomène à étudier. Ainsi, à partir d'une même donnée satellitale, deux utilisateurs ayant des thèmes de recherche différents utiliseront le même outil de classification mais y introduiront des attributs différents. Ceux-ci sont les données brutes (soit les différents canaux) et/ou des néo-canaux : indices ratio (rapport de deux canaux bruts) ou texturaux. Il faut souligner que les images ratios permettent de réduire les effets perturbateurs du rayonnement solaire et des caractéristiques atmosphériques. Ils suppriment les variations de brillance imputables à la topographie et renforcent les variations spectrales. Le facteur albédo disparaît pourtant et deux objets ayant un même ratio mais des albédos différents peuvent être confondus. Il est donc conseillé d'utiliser simultanément les images brutes et les ratios. Des indices texturaux simples à calculer tels que le mode local ou la variance 
locale dans une fenêtre $3 \times 3$ ou $5 \times 5$, peuvent améliorer visiblement les résultats (Hakdaoui, 1991).

L'introduction de classes intermédiaires dans une classification est une notion qui permet de distinguer des pixels purs (correspondant à des zones homogènes) de pixels mélangés, appelés aussi pixels hybrides (correspondant à des zones de transition). Ceux-ci constituent une classe à part dont le centre initial aura été introduit par l'utilisateur (classification supervisée) : mais cette approche est délicate en raison de la difficulté de définir spatialement les unités. En effet les milieux littoraux sont par nature très dynamiques et les zones de transition y sont particulièrement instables. Ces pixels mixtes peuvent aussi se trouver dans une classe rejet si une fonction de décison d'appartenance à une classe a été utilisée (fig. 1). Elle permet, à partir d'un seuil fixé, de rejeter les pixels dont la proximité à la classe la plus proche n'est pas satisfaisante (Boujemaa et al., 1994 ; Caillol et al., 1994).

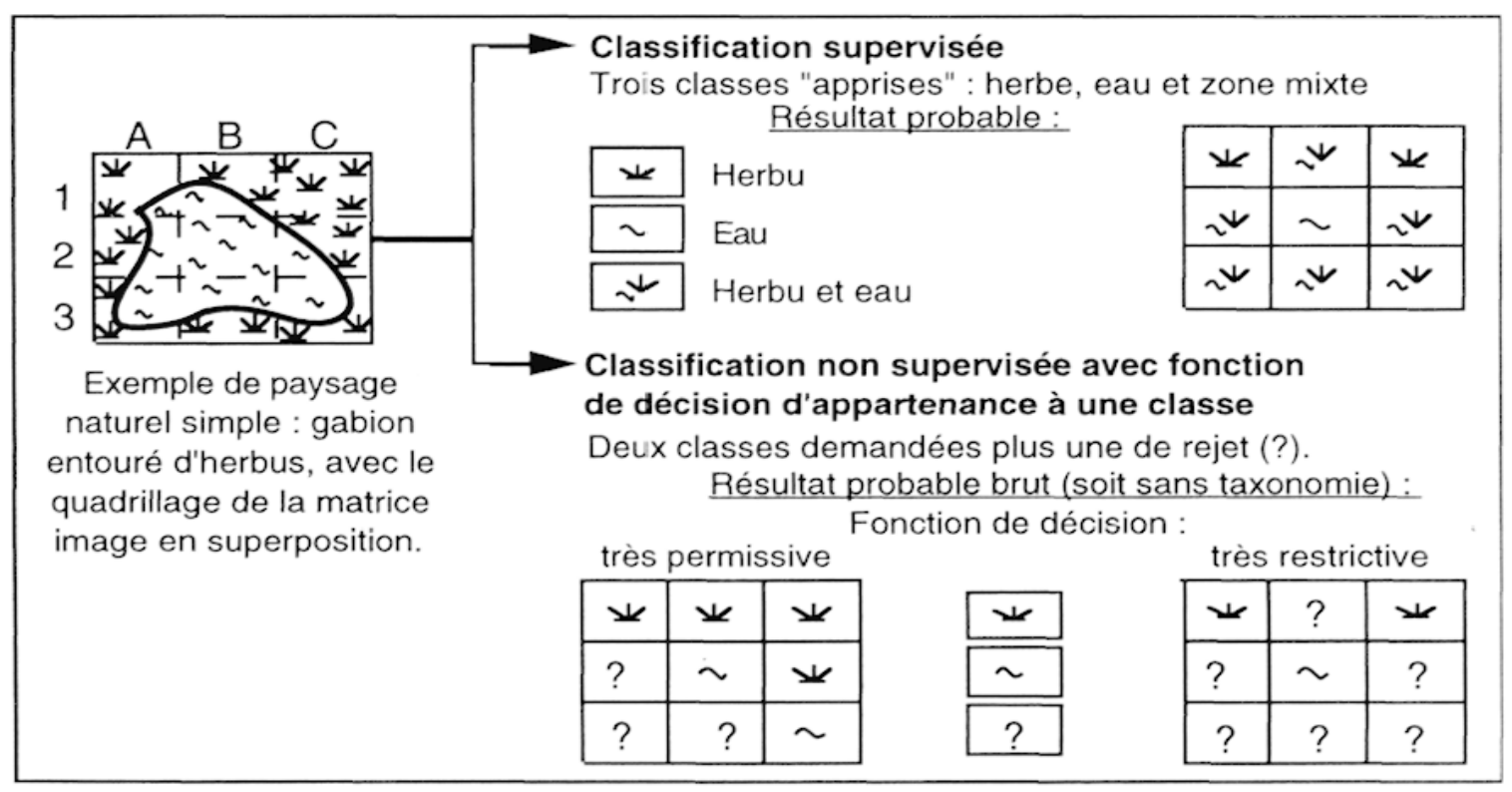

FIG. 1. - Classification supervisée et non supervisée avec fonction de décision d'appartenance, sur un paysage schématisé d'un wadden.

\section{B) LES DÉTECTIONS DE GRADIENTS}

Un gradient est une rupture des valeurs numériques. La mise en évidence des discontinuités est généralement réalisée par la convolution de filtres morphologiques (Prewit, Laplacien, Sobel...) dont certains ne considèrent qu'une orientation pré-définie : filtre diagonal, horizontal (Bonn et Rochon, 1992).

L'image résultante (image-gradient) est le plus souvent seuillée afin de ne garder que les valeurs élevées significatives des discontinuités nettes qui marquent les limites des unités géographiques. C'est pourquoi l'expression détection de contours est souvent utilisée (Haese-Coat et al., 
1994). Par exemple, considérant une parcelle d'herbe au milieu de sols labourés, la microtexture de l'herbe caractérise cet objet tandis qu'une image-gradient seuillée donnera l'extension de celui-ci. Dans un espace intertidal vaseux où évoluent des chenaux de marée, la texture sera probablement semblable des deux côtés d'un chenal tandis qu'il sera possible de localiser ses berges par une détection de gradients.

La recherche de gradients sur une donnée issue d'une classification, correspond, de fait, à l'extraction des contours de classes. Sur une image quelconque, canal brut ou néo-canal, le résultat de la convolution est souvent une image surchargée de pixels isolés. Il est alors possible de les éliminer par filtrage mais beaucoup plus délicat de joindre les segments pertinents pour déterminer les contours des objets.

La photo-interprétation (technique manuelle) reste très utilisée car elle est encore la méthode qui donne les résultats les plus satisfaisants aux yeux de l'expert qui met tout son jugement dans ses tracés et s'assure que le résultat soit dépourvu de bruit (pixels isolés ou segments sans rapport avec sa thématique).

\section{C) LES MODĖLES}

Un modèle, en télédétection, est l'expression d'une relation qui existe entre une donnée satellitale et un phénomène physique à étudier (exemple : réflectance et hauteur de la tranche d'eau). Ainsi le lien entre les longueurs d'onde utilisées par le modèle et la thématique recherchée doit être sans ambiguîté. Dans les zones subtidales, chaque longueur d'onde du rayonnement solaire présente un maximum de pénétration. Les profondeurs supérieures à celui-ci s'expriment par un plancher radiométrique (dit aussi radiométrie infinie : Rinf de la fig. 2). Les modèles sont donc limités dans ce domaine par ce seuil qui varie surtout en fonction des longueurs d'onde (sachant que les plus courtes sont les plus pénétrantes) et la qualité des eaux (ainsi plus elles sont troubles, plus vite la limite de la relation radiométrie - profondeur sera atteinte) (fig. 2).

$\mathrm{S}$. Andrefouet (1990) considère deux approches de modélisation pour l'extraction de l'information pseudo-bathymétrique en imagerie optique. La première est une approche physique qui cherche à prendre en compte l'ensemble des paramètres qui affectent la radiométrie en s'affranchissant de mesures sur le terrain. Elle requiert une bonne connaissance de l'environnement lors de la prise de vue. Les modèles de ce type sont fondés sur la relation :

$\mathrm{R}_{(\mathrm{i})}=\mathrm{Rs}_{(\mathrm{i})}+\mathrm{K}_{(\mathrm{i})} * \mathrm{RB}_{(\mathrm{i})} * \exp \left(\mathrm{kd}_{(\mathrm{i})} * \mathrm{fz}\right)$ où $\mathrm{i}$ est le numéro du canal, $\mathrm{R}_{(\mathrm{i})}$ la radiométrie mesurée, $\mathbf{R s}_{(\mathrm{i})}$ la radiométrie infinie (en eau profonde), $\mathrm{Kd}_{(\mathrm{i})}$ le coefficient dépendant de la rugosité de surface, de la transmittance de l'atmosphère..., $\mathrm{RB}_{(\mathrm{i})}$ le coefficient de réflexion du fond selon sa nature, $\mathrm{kd}_{(\mathrm{i})}$ le coefficient d'atténuation de l'eau de mer et $\mathrm{fz}$ le facteur géométrique $(\mathrm{fz}=(\mathrm{z}+\operatorname{cosec}[e])$ où $\mathrm{z}$ est la profondeur et $e$ l'angle de réfraction, dépendant de l'élévation du soleil et du coefficient de réfraction) 
(Andrefouet, 1990). La seconde approche est dite «de terrain» car elle s'appuie sur des mesures in situ et s'affranchit des paramètres physiques. Le principe est d'établir une relation mathématique entre radiométrie et bathymétrie d'après les points de calage sélectionnés, puis de l'étendre à l'ensemble de l'image.

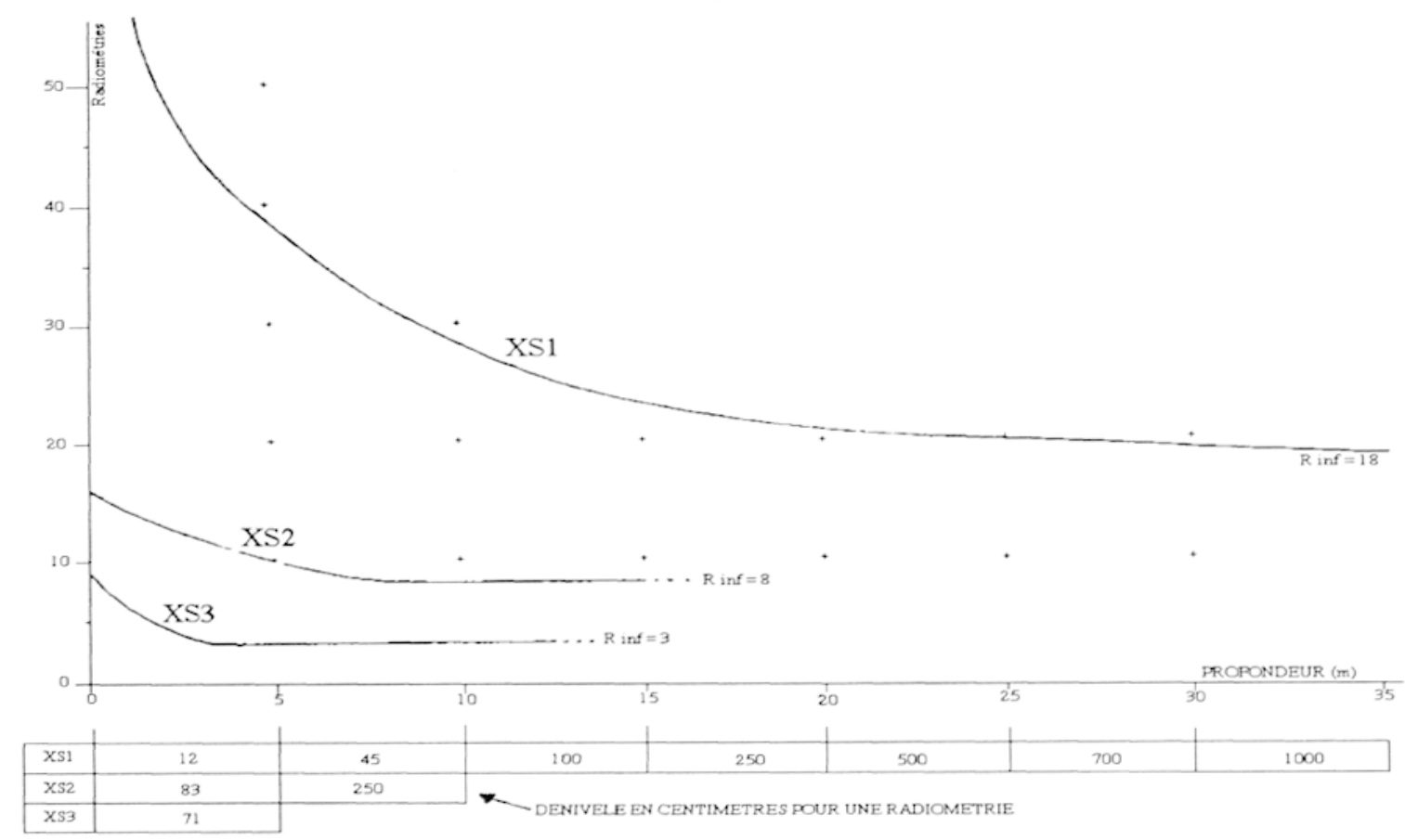

FIG. 2. - Radiométries de HRV-SPOT et profondeurs dans les eaux du Pacifique. (Garlan, 1989).

Finalement, l'approche physique s'avère difficile à mettre en œuvre car il est très rare de connaître précisément l'ensemble de ces coefficients ; il apparaît que l'approche terrain est la plus répandue et donne d'assez bons résultats.

\section{II. - LES MÉTHODES POUR ÉTABLIR DES SPATIO-CARTES PRÉCISES}

\section{A) CARTOGRAPHIE DE LA ZONE ÉMERGÉE DE LA BAIE DU MONT SAINT-MICHEL PAR CLASSIFICATION}

Les images à marée basse ou en début de flot sont à privilégier afin de limiter l'effet perturbateur de la teneur en eau des sédiments de l'estran. L'information radiométrique contenue dans un pixel est utilisée afin de définir, toujours avcc un degré d'incertitude, l'objet géographique correspondant. Pour la végétation seule, un indice de végétation normalisé tel que : $(P I R-R) /(P I R+R)$ où PIR est le proche infra-rouge et $R$ le 
rouge, une fois discrétisé, permet d'obtenir des classes illustrant le taux de recouvrement par la végétation chlorophyllienne. Pour l'étude des végétaux, la saison a une influence déterminante.

L'exemple présenté ici concerne la baie du Mont-Saint-Michel. La donnée a été acquise par le satellite SPOT en mode multispectral le 9 juin 1986 à 10 h 30 TU. Le capteur HRV fournit trois images dont deux dans le visible : Xsl $(500-590 \mathrm{~nm})$ et Xs2 $(610-680 \mathrm{~nm})$ et une dans le proche infrarouge : Xs3 (790-890 $\mathrm{nm})$.

La taxonomie d'une classe étant définie par la signature spectrale de son centre, les courbes de réflectance de quelques objets typiques de la baie du Mont-Saint-Michel sont présentées dans la fig. 3. Il y apparaît que la composition du sédiment entre en compte ainsi que son état hygrométrique, sa granulométrie et l'existence d'une couverture végétale.

Tandis que les sables ont une réponse forte et croissante avec la longueur d'onde, les vases présentent une courbe basse et plate. Ceci s'explique surtout par trois facteurs.

- La couleur du sédiment : les vases grises réfléchissent moins que les sables souvent plus clairs.

- Leur granulométrie ; les vases plus compactes ont des teneurs en eau supérieures à celles des sables dont l'essorage sur l'estran est d'autant plus rapide qu'il s'agit de sables grossiers. Or l'eau absorbe de plus en plus les rayonnements du visible au proche infrarouge (fig. 4).

- leur teneur en carbonates (coquilles entières ou en débris) augmente la réflectance, surtout dans le domaine du proche infrarouge (fig. 4).

Par ailleurs, la signature spectrale d'un sédiment est affectée par la présence d'une couverture végétale plus ou moins discontinue. Ainsi les diatomées sur les vases de l'estran provoquent une chute de la réflectance vers $680 \mathrm{~nm}$ suivie par une remontée de la courbe.

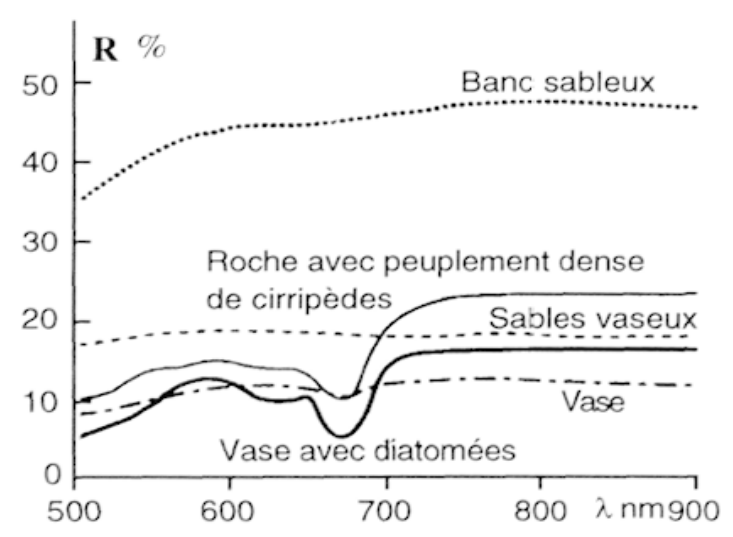

FIG. 3. - Exemple de signatures spectrales de sédiments de la baie du Mont-Saint-Michel (d'après Guillaumont, 1989).

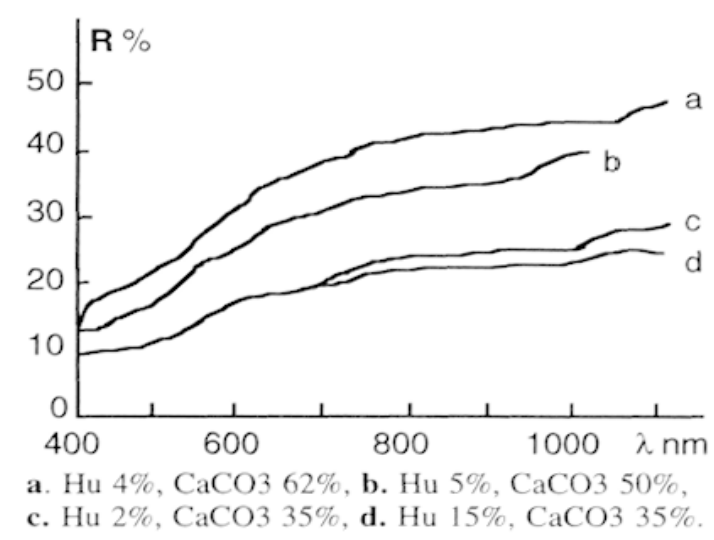

Fig. 4. - Influence des teneurs en carbonate et en eau sur une signature d'un sable (d'après Zbinden, 1987). 
La fig. 5 est le résultat des traitements effectués qui peuvent se résumer en quatre points :

1) Extraction des zones littorales, le reste, constitué essentiellement des parties terrestres, est masqué.

2) Extraction d'une classe Eau libre comprenant la zone infratidale et les eaux saumâtres des chenaux. Cette opération est réalisée par un seuillage de la donnée Xs3 la plus sensible à l'humidité. Le seuil choisi correspond à la radiométrie maximale du premier mode de l'histogramme centré sur la radiométrie infïnie (Rinf).

3) Classification non supervisée par nuées dynamiques avec cinq classes sur les canaux bruts (Xs1, Xs2 et Xs3) après avoir exclu les pixels du masque ou de la classe Eau libre.

4) Élaboration de la taxonomie après interprétation des signatures des barycentres des classes.

Finalement la fig. 5 présente une cartographie synthétique de la baie du Mont-Saint-Michel au moment de l'acquisition de l'image. La limite instantanée de la mer est une isobathe et les différences d'humidité des sédiments de la zone intertidale émergée renseignent sur la topographie et la localisation des chenaux de marée. Les sables de haute plage et les bancs coquilliers supratidaux s'individualisent parfaitement ainsi que les zones colonisées par la végétation halophyte. Ainsi, au-delà de la représentation immédiate de l'estran, il est envisageable d'étudier la dynamique du front des herbus et les étapes de l'ensablement à condition que la fréquence des acquisitions par le satellite le permette.

\section{B) CARTOGRAPHIE DE LA MORPHOLOGIE DES FONDS PAR DÉTECTION DE GRADIENTS}

Les ondes visibles ou radar ne peuvent atteindre les fonds dans des eaux troubles. En conséquence pour apprécier ceux-ci force est de passer par une voie indirecte qui est l'état de surface de la mer. Car les fonds ont une incidence en surface sur les très courtes vagues; l'image du champ de celles-ci reflète alors la morphologie du fond. Ces vagues, dites d'ultragravité, de longueur d'onde centimétrique ou décimétrique, sont levées instantanément par le vent à la surface de l'eau. D'après des études statistiques, l'IFP a défini les limites de perception du fond par les ondes d'ultra-gravité. Le vent de surface doit être compris entre 10 et 30 nœuds et les courants entre 0,7 et 2,2 nœuds. Quant à la direction du vent et des courants, elles doivent être opposées pour lever ces vagues. Les fonds doivent être couverts par une tranche d'eau de 50 à 80 mètres au maximum (Wadsworth et Piau, 1984). Par ailleurs, les déclivités doivent être importantes afin d'entraîner, en conjugaison avec les courants, des ondes d'ultra-gravité en surface car la qualité de perception de ces objets est moins liée à leur amplitude qu'aux ruptures et inversions de pentes qu'ils créent. Les images radar semblent les plus appropriées pour ce type 


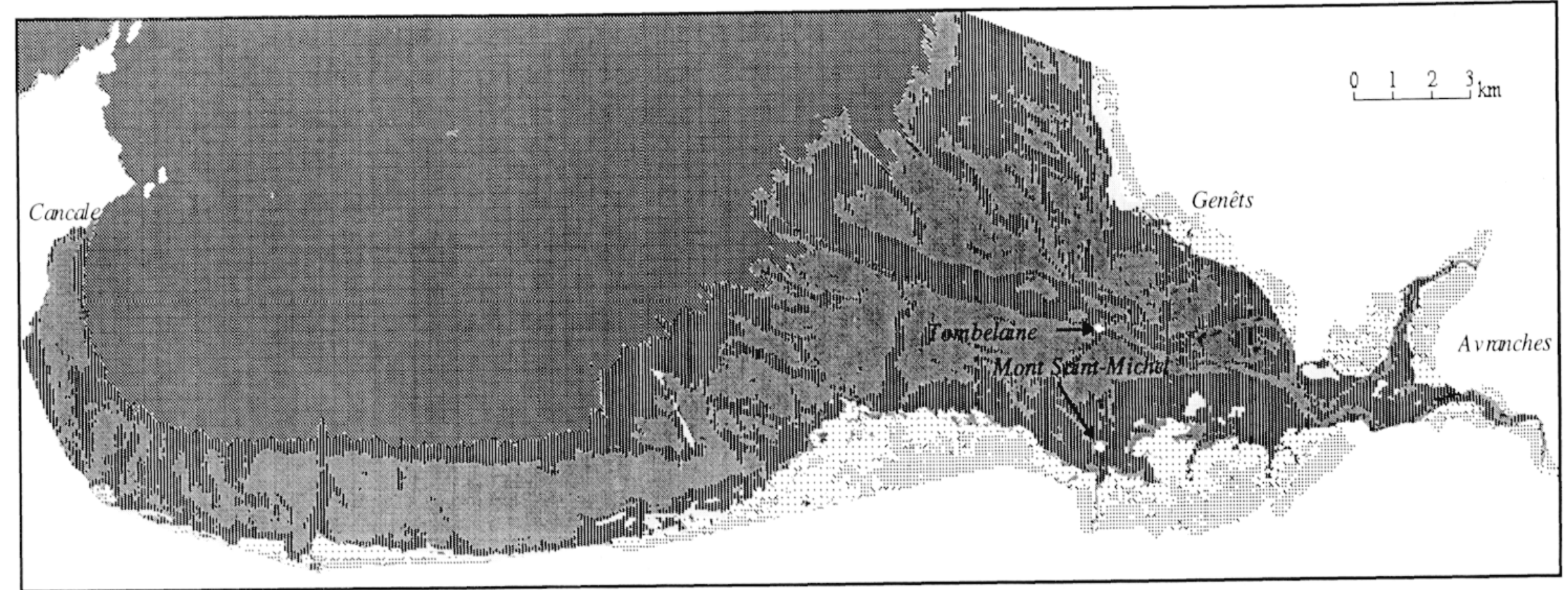

Couvert végétal à prédominance de graminées (vieux schorres et dunes).

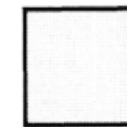

Sables fins de haute plage et bancs coquilliers.

Sablons.

Herbu à peuplement d'obiones,

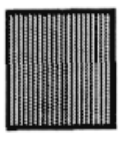

Tangues et crème de vase.

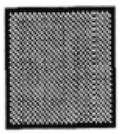

Mer. de salicornes et de soudes.

FiG. 5. - La baie du Mont-Saint-Michel le 09/06/1986 à 10 h 30 TU, 3 h 30 après la pleine mer (cœf. 70). 
d'approche dans la mesure où elles mettent en exergue les rugosités de surface.

Si ces limites ne permettent pas ce type d'étude en baie du Mont-SaintMichel, une étude de hauts fonds marins a été menée sur le littoral du Nord-Pas-de-Calais (Lapie, 1984). Le but était de retrouver sur une image radar du satellite Seasat, la morphologie des dunes submergées cartographiées par ailleurs sur des minutes bathymétriques. Ces grandes dunes sont parallèles au courant dominant et comprennent des petites vagues de sable perpendiculaires à l'axe général qui sont créées par des courants pouvant atteindre 3 nouds par endroits. À partir des minutes de sonde, des profils transversaux et longitudinaux ont été réalisés. Sur l'image radar des profils sont effectués sur une zone remarquable par ses petites vagues. Sur cette donnée satellitale, les ondes d'ultra-gravité ont permis de retrouver toutes les dunes positionnnées sur les relevés bathymétriques.

Ainsi, si toutes les conditions océanographiques et météorologiques quantifiées par l'IFP sont réunies, l'essentiel des reliefs sous-marins peut se retrouver sur une image radar via des phénomènes induits en surface. Ceci permet d'envisager entre autre le suivi des déplacements et des évolutions morphologiques de bancs de sable.

L'étude de la réfraction de la houle permet aussi d'appréhender indirectement la morphologie des fonds marins. Fr. Cuq (1984) a présenté une méthode de détection automatique des champs de houle à partir des données du radar SAR de Seasat dans le Pertuis breton. A partir de la donnée satellitale deux néo-canaux sont calculés représentant l'intensité du gradient et l'orientation des trains de houle. Sur cette dernière, un seuillage permet d'obtenir les crêtes de vagues d'une seule orientation. Tandis qu'un seuillage de l'image gradient permet de repérer les zones de perturbations qui signalent des accidents topographiques du fond.

\section{C) CARTOGRAPHIE DE LA PSEUDO-BATHYMÉTRIE PAR MODĖLE} (APPROCHE DE TERRAIN)

Les images optiques permettent, dans une certaine mesure, de quantifier les hauteurs de la tranche d'eau. L'inconvénient essentiel est la turbidité des eaux puisqu'on tente de corréler directement radiométrie et bathymétrie. Chaque pixel est traité indépendamment de ses voisins afin de lui attacher une hauteur d'eau. Ensuite, ils sont éventuellement rassemblés en classes homogènes.

Th. Garlan (1989) s'est affranchi totalement des paramètres physiques pour l'élaboration de spatio-cartes marines en utilisant la relation $Z=A$ * $L N\left(R_{(i)}-\operatorname{Rinf}_{(i)}+B^{*} L N\left(R_{(j)}-\operatorname{Rinf}_{(j)}\right)+C\right.$ où $Z$ est la profondeur, i et $j$ les numéros des canaux Xsl et Xs 2 de Spot, $R$ la radiométrie mesurée, Rinf la radiométrie en eau profonde. A et $\mathrm{B}$ sont les coefficients calculés à partir des points de calage par une régression et $C$ une constante. Ce modèle prend en entrée une image Spot prétraitée, c'est-à-dire la donnée brute ayant subi un masquage des terres, des nuages et de leur ombre puis une 
correction de l'effet de colonnage du capteur sur les parties maritimes. L'application s'effectue sur les littoraux coralliens français du Pacifique où les eaux sont très claires et les fonds de nature homogène. Le coefficient d'absorption de l'eau et celui dû à la nature des fonds sont supposés identiques en tout point de l'image : ils sont pris en compte une fois pour toutes par les paramètres $\mathrm{A}, \mathrm{B}$ et $\mathrm{C}$ de la relation. Pourtant, les pinacles coralliens qui représentent des exceptions bien individualisées par des réflectances plus faibles que leur environnement engendrent une surestimation importante de leur profondeur représentant en cela un danger pour la navigation. Ils montrent la limite de validité de la méthode qui pourrait être enrichie par une prise en compte du canal Xs3 pour les très petits fonds (fig. 2) (Garlan, 1989). Finalement le modèle est validé par l'analyse des écarts quadratiques moyens aux points de calcul. Les résultats sont plus ou moins satisfaisants et dépendent beaucoup du site considéré. En eaux claires, Th. Garlan obtient une erreur de l'ordre de $10 \%$ pour des fonds inférieurs à $-5 \mathrm{~m}$ et supérieure à $20 \%$ en-deçà en raison des difficultés de repérage des pinacles.

L'intérêt d'un modèle pour ce type de problématique décroît rapidement lorsque la turbidité augmente car cette variable est un facteur très limitant pour l'extraction de l'information pseudo-bathymétrique d'après des données satellitales. Dans les eaux troubles des passes du bassin d'Arcachon, l'étude menée par V. Bricourt et J.-M. Froidefond (1993) à partir d'images Spot montre que la profondeur maximale au-delà de laquelle les radiométries n'évoluent plus (Rinf) est limitée au mieux à 8 mètres.

\section{CONCLUSION}

Les tableaux I et II résument les méthodes et leurs limites par rapport à un objectif cartographique précis défini préalablement pour les zones intertidale et subtidale.

TABLEAU I. - Objectifs cartographiques et télédétection en zone subtidale.

\begin{tabular}{|l|l|}
\hline \multicolumn{1}{|c|}{$\begin{array}{c}\text { OBJECTIFS } \\
\text { CARTOGRAPHIQUES }\end{array}$} & \multicolumn{1}{c|}{$\begin{array}{c}\text { LIMITES ET PROPOSITIONS } \\
\text { DE TRAITEMENT DE LA DONNÉE SATELLITALE }\end{array}$} \\
\hline $\begin{array}{l}\text { Bathymétrie } \\
\text { Appréciation quantitative }\end{array}$ & $\begin{array}{l}\text { Par modélisation, d'après des images dans les plus courtes } \\
\text { longueurs d'onde, dans des eaux calmes et claires avec des } \\
\text { fonds de nature homogène sinon, il y a risque d'interférence } \\
\text { avec d'autres facteurs (teneur en M.E.S. ou nature des fonds). }\end{array}$ \\
\hline $\begin{array}{l}\text { Morphologie des fonds } \\
\text { Appréciation qualitative }\end{array}$ & $\begin{array}{l}\text { Sur une image radar ou optique, par extraction de gradients } \\
\text { marquant les crêtes de vague (ondres d'ultra-gravité ou champ de } \\
\text { houle). Les conditions océanographiques et météorologiques } \\
\text { doivent être réunies pour permettre aux reliefs submergés } \\
\text { d'induire des perturbations en surface. }\end{array}$ \\
\hline Teneur en M.E.S. & $\begin{array}{l}\text { Par classification non supervisée pour avoir une idée qualitative } \\
\text { de la répartition des M.E.S. Par modélisation, mais les points de } \\
\text { calage doivent être pris en concomitance avec l'acquisition par le } \\
\text { satellite (surtout dans les baies ou estuaires à marée importante). }\end{array}$ \\
\hline
\end{tabular}


TABLEAU II. - Objectifs cartographiques et télédétection en zone intertidale et supratidale.

\begin{tabular}{|l|l|}
\hline \multicolumn{1}{|c|}{$\begin{array}{c}\text { OBJECTIFS } \\
\text { CARTOGRAPHIQUES }\end{array}$} & \multicolumn{1}{c|}{$\begin{array}{c}\text { LIMITES ET PROPOSITIONS } \\
\text { DE TRAITEMENT DE LA DONNÉE SATELLITALE }\end{array}$} \\
\hline $\begin{array}{l}\text { Végétaux de la partie } \\
\text { supratidale }\end{array}$ & $\begin{array}{l}\text { Par seuillage d'un indice de végétation ou par classification, } \\
\text { supervisée ou non, d'images optiques (visible et proche } \\
\text { infrarouge). L'état de végétaux selon la saison est à prendre } \\
\text { en compte dans l'interprétation finale et le choix des zones } \\
\text { d'apprentissage. }\end{array}$ \\
\hline Sédiments & $\begin{array}{l}\text { Par classification, sur des images optiques à marée basse ou en } \\
\text { début de flot afin que l'humidité soit la plus faible. Il est possible } \\
\text { de tenter d'améliorer le résultat en ajoutant des néocanaux } \\
\text { comme la variance locale pour la rugosité de surface. }\end{array}$ \\
\hline $\begin{array}{l}\text { Chenaux de marée } \\
\text { sur l'estran }\end{array}$ & $\begin{array}{l}\text { Par photo-interprétation, sur une image à mi-marée où les } \\
\text { chenaux sont encore remplis d'eau. Éventuellement un seuillage } \\
\text { pourra donner satisfaction. }\end{array}$ \\
\hline
\end{tabular}

Finalement, la description sédimentologique et morphologique d'un estran via des données de satellites radars ou optiques conduit à mettre en œuvre des techniques très variées. De plus, cela nécessite de nombreuses données exogènes qui sont prises sur le terrain ou relevées sur des documents. Dans tous les cas, celles-ci doivent être le plus proche possible de la date d'acquisition des scènes car les littoraux sont parmi les milieux physiques les plus dynamiques.

\section{BIBLIOGRAPHIE}

ANDREFOUET (S.), 1990. - Topographie et bathymétrie; méthodes et outils. Application à la représentation tridimensionnelle d'un atoll et de son lagon. Rapport de stage à l'IFREMER, $60 \mathrm{p}$.

Bonn (F.), Rochon (G.), 1992. - Précis de télédétection, l-Principes et méthodes. Presses de l'Université du Québec, Sillery, 486 p.

Boujemaa (N.), Stamon (G.), Gagalowicz (A.), 1994. - Modélisation floue pour la segmentation d'images. Actes du $9^{c}$ congrès « Reconnaissance des formes et intelligence artificielle », 11-14 janv., Paris, pp. 163-173.

Bricourt (V.), Froidefond (J.-M.), 1993. - La télédétection : un outil pour la bathymétrie côtière . Bulletin Inst. Géol. Bassin Aquitaine, $\mathrm{n}^{\circ}$ 53, pp. 199-207.

Caillol (H.), Hillion (A.), Pieczynski (W.), 1994 - Segmentation contextuelle non supervisée d'images utlisant une modélisation statistique floue. Actes du $9^{\mathfrak{c}}$ congrès « Reconnaissance des formes et intelligence artificielle », 11-14 janv., Paris, pp. 145151.

Charron (C.), Lointier (M.), Prost (M.-T.), Rudant (J.-P.), 1990. - Etude multitemporelle du littoral et des estuaires guyanais utilisant l'imagerie Spot et Landsat. Actes du Symposium "Evolution des littoraux de Guyane et de la zone caraïbe méridionale pendant le Quaternaire ", 9-14 nov., Cayenne (Guyane), ed. ORSTOM, pp. 61-71.

CUQ (Fr.), 1984. - Interprétation automatique des champs de vagues à partir des données du radar SAR de Seasat 1 Photo-interprétation, ${ }^{\circ} 4$, pp. $1-7$.

Equipe Fralit, 1977. - Télédétection du littoral océanique de la France. ENS Montrouge-EPHE, $310 \mathrm{p}$. 
GaRlan (Th.), 1989. - Cartographie spatiale du littoral corallien : topographie et bathymétrie. Rapport d'étude de l'EPSHOM, $\mathrm{n}^{\circ} 006 / 89,25$ p.

GUILlaUmONT (B.), 1989. - Utilisation de l'imagerie satellitale pour les comparaisons spatiales et temporelles en zone intertidale. Actes du Colloque ECSA 19 «Milieux estuariens et littoraux : intercomparaisons spatiales et temporelles », Caen, pp. 63-68.

Haese-Coat V., KPalma K., Ronsin J., 1994. - Apport comparatif de la texture en segmentation d'image SPOT, Application à la forêt de Paimpont. Photointerprétation, 1-2, pp. 49-54.

HAKDAOUI (M.), 1991. - Apport de l'analyse texturale à l'interprétation géologique et géomorphologique d'images digitales. Thèse de l'Univ. Paris VI, 82 p.

LAPIE (E.), 1984. - Etudes des hauts fonds marins à partir des images radar. Rapport de l'Institut Français du Pétrole, $\mathrm{n}^{\circ} 32560,10 \mathrm{p}$.

Wadsworth (A.), Piau (P.), 1984. - Morphologie radar de fonds marins. Revue de l'Institut Français du Pétrole, n ${ }^{\circ} 39,2$, pp. 161-174.

ZBINDEN (R.M.), 1987. - Analyse spectrale des matières en suspension dans l'eau de mer et des sédiments d'estran. Rapport d'actions thématiques programmées ATP "Télédétection spatiale 1985 », ENS-Montrouge, 14 p.

\section{REMERCIEMENTS}

Ce travail est cofinancé par le Conseil Régional de Basse-Normandie et le BRGM. 